\title{
DETECTABILITY OF EXOPLANET PERIASTRON PASSAGE IN THE INFRARED
}

\author{
Stephen R. Kane and Dawn M. Gelino \\ NASA Exoplanet Science Institute, Caltech, MS 100-22, 770 South Wilson Avenue, Pasadena, CA 91125, USA; skane@ipac.caltech.edu \\ Received 2011 March 31; accepted 2011 August 5; published 2011 October 14
}

\begin{abstract}
Characterization of exoplanets has matured in recent years, particularly through studies of exoplanetary atmospheres of transiting planets at infrared wavelengths. The primary source for such observations has been the Spitzer Space Telescope but these studies are anticipated to continue with the James Webb Space Telescope. A relatively unexplored region of exoplanet parameter space is the thermal detection of long-period eccentric planets during periastron passage. Here we describe the thermal properties and albedos of long-period giant planets along with the eccentricities of those orbits which allow them to remain within the habitable zone. We further apply these results to the known exoplanets by calculating temperatures and flux ratios for the IRAC passbands occupied by warm Spitzer, considering both low and high thermal redistribution efficiencies from the perspective of an observer. We conclude with recommendations on which targets are best suited for follow-up observations.
\end{abstract}

Key words: planetary systems - techniques: photometric

\section{INTRODUCTION}

As the number of known exoplanets continues to rise at a steady pace, their diversity appears to only increase. For instance, transiting exoplanets have allowed us to access properties of exoplanet atmospheres through observations at infrared (IR) wavelengths during secondary eclipse and atmospheric absorption during primary transit (Agol et al. 2010; Deming et al. 2007). Further opportunities for atmospheric studies have arisen through the detection of phase variations of such planets as HD 189733b (Knutson et al. 2009a) and HD 149026b (Knutson et al. 2009b). These phase variations have also been detected for non-transiting planets, including $v$ And $\mathrm{b}$ (Crossfield et al. 2010; Harrington et al. 2006) and HD 179949b (Cowan et al. 2007). Significant constraints have been placed on the planetto-star flux ratio for HD 217107b through ground-based near-IR observations by Cubillos et al. (2011).

Studies thus far have largely been directed toward shortperiod planets which are expected to have an a priori high effective temperature. In particular, this exclusivity results from a relatively high thermal flux required from the planet in order for the signature to be detectable. The exception to this is the planet HD 80606b, with a highly eccentric orbit $(e=0.93)$ and a period of $\sim 111$ days, detected by Naef et al. (2001). Subsequent Spitzer observations by Laughlin et al. (2009), as well as the detection of the secondary eclipse of the planet, were used to measure the out-of-eclipse variations and estimate the radiative time constant at $8 \mu \mathrm{m}$. As pointed out by Barbieri et al. (2007), ambiguous measurements of radiative time constants have prevented a consensus on expected planet-wide flow patterns for short-period planets.

Recent three-dimensional models of planetary atmospheres (Barman et al. 2005; Dobbs-Dixon et al. 2010; Fortney et al. 2010; Koskinen et al. 2007; Lewis et al. 2010; Madhusudhan \& Seager 2010; Rauscher \& Menou 2010; Showman et al. 2008; Sudarsky et al. 2005; Thrastarson \& Cho 2010) have made significant progress in deriving the underlying atmospheric physics which drives the thermal properties and zonal winds in exoplanetary atmospheres. Highly eccentric planets such as HD $80606 \mathrm{~b}$ provide a means to explore atmospheric properties in a different regime of orbital parameter space since the heating of the atmosphere during periastron passage can be sufficient to produce a detectable signal (Cowan \& Agol 2011a). The possibility of investigating eccentric planets at optical wavelengths has previously been explored by Kane \& Gelino (2010, 2011). This observing window for periastron passage is brief and requires a reasonable understanding of the orbital parameters (Kane et al. 2009).

This paper describes the predicted thermal changes for exoplanets in highly eccentric orbits. This study is primarily motivated from an observer's point of view and is mostly concerned with planets which are not known to produce either a secondary eclipse or primary transit. We derive analytical expressions for the albedos based upon theoretical models and calculate the effective temperatures and flux ratios, taking into account the thermal heat redistribution and radiative time constant. These calculations are applied to the known exoplanets for which orbital parameters measured from radial velocity data are available. We explore the dependencies of the planetary effective temperatures on eccentricity and orbital period and determine the percentage of the orbits which are spent in their respective habitable zones (HZs). We finally calculate predicted maximum flux ratios during periastron passage, discuss the effect of spots on detections, and propose potential targets for Spitzer and James Webb Space Telescope (JWST) observations.

\section{PLANETARY EFFECTIVE TEMPERATURES}

In this section, we outline the basic assumptions used to calculate the planetary effective temperatures throughout the remainder of the paper. These assumptions have been deliberately introduced to be fairly broad since the intention is to encompass a variety of planets to produce a first-order approximation of the global distribution of thermal signatures and detectability. These were designed in such a way as to produce testable limits on the flux ratios at periastron passage.

We begin with the luminosity of the host star, which is approximated as

$$
L_{\star}=4 \pi R_{\star}^{2} \sigma T_{\mathrm{eff}}^{4},
$$

where $R_{\star}$ is the stellar radius, $T_{\text {eff }}$ is the stellar effective temperature, and $\sigma$ is the Stefan-Boltzmann constant. In cases where the radius of the star is not available from direct measurements, we estimate the radius from the derived values 
of the surface gravity $\log g$ using the relation

$$
\log g=\log \left(\frac{M_{\star}}{M_{\odot}}\right)-2 \log \left(\frac{R_{\star}}{R_{\odot}}\right)+\log g_{\odot},
$$

where $\log g_{\odot}=4.4374$ (Smalley 2005).

As described by Knutson et al. (2009b), we can approximate the effective temperature of a planet, $T_{p}$, as a blackbody. This approximation will deviate slightly from the true temperature depending upon albedo, atmospheric properties, and internal heating. Assuming that the atmosphere is $100 \%$ efficient at redistributing heat around the planet, the planetary equilibrium effective temperature is given by

$$
T_{p}=\left(\frac{L_{\star}(1-A)}{16 \pi \sigma r^{2}}\right)^{\frac{1}{4}}
$$

where $A$ is the spherical (Bond) albedo and $r$ is the star-planet separation. In this case, the surface is uniformly bright and thus there will be no observable phase function at IR wavelengths. However, if the atmosphere is inefficient with respect to heat redistribution, this will lead to a hot day side for the planet where the effective temperature is

$$
T_{p}=\left(\frac{L_{\star}(1-A)}{8 \pi \sigma r^{2}}\right)^{\frac{1}{4}}
$$

where there will be a resulting phase variation as the planet orbits the star. The generalized form for the planetary effective temperature is thus

$$
T_{p}=\left(\frac{L_{\star}(1-A)}{(1+\eta) 8 \pi \sigma r^{2}}\right)^{\frac{1}{4}}
$$

where $\eta$ is the atmospheric heat redistribution efficiency with a value ranging between 0 and 1 .

For a typical hot-Jupiter scenario, the star-planet separation is assumed to be the same as the semimajor axis, $a$, since these are usually circular orbits. However, the star-planet separation for eccentric planets has the following form:

$$
r=\frac{a\left(1-e^{2}\right)}{1+e \cos f}
$$

where $e$ is the orbital eccentricity and $f$ is the true anomaly. Thus, the eccentricity of a planetary orbit introduces a time dependency to the effective temperature of the planet. In the absence of atmospheric effects (see Section 3), the temperature of the planet may be examined as indicated in Figure 1, where the dependence on star-planet separation is shown for two example spectral class targets. For a given spectral type, the difference between $0 \%$ and $100 \%$ heat redistribution efficiency can lead to a $\sim 20 \%$ adjustment in the temperature calculation. However, $r$ is clearly dominant over $\eta$ at all separations. This means that the planetary temperature, and thus the flux ratio, is more dependent upon the orbital properties (which are well determined) than on the atmospheric dynamics (which are generally unknown). This is elaborated upon in the following section.

\section{ATMOSPHERIC PROPERTIES}

Here we discuss the major atmospheric properties which directly influence planetary thermal signatures.

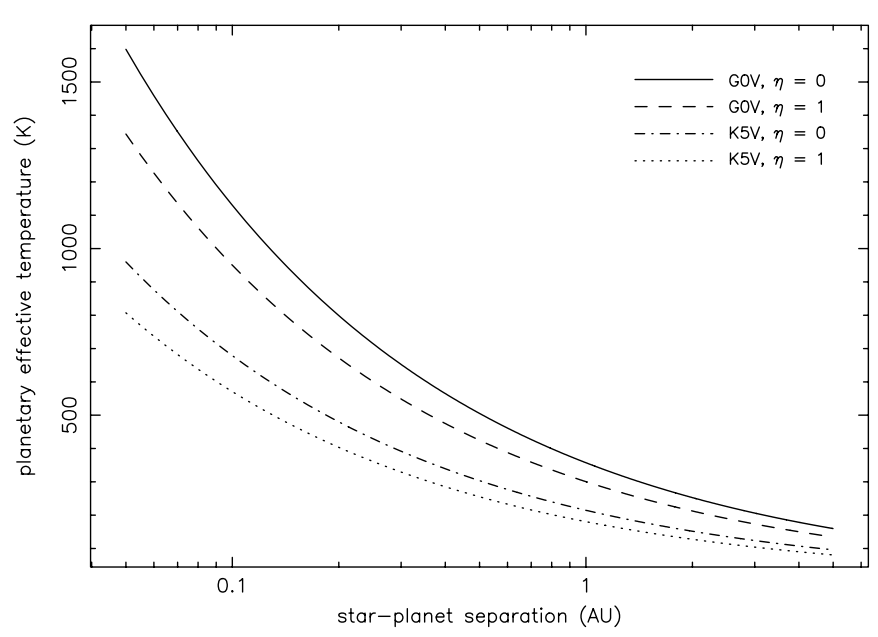

Figure 1. Dependence of planetary effective temperature on star-planet separation for inefficient $(\eta=0)$ and efficient $(\eta=1)$ heat redistributions. This is calculated for G0V and K5V host stars and shows that the scale of the temperature variations is dominated by the star-planet separation and not by the heat redistribution efficiency.

\subsection{Spherical Albedo}

The spherical albedo of planetary atmospheres beyond the hot-Jupiter regime is less understood at IR wavelengths $(\sim 10 \mu \mathrm{m})$ than it is at optical wavelengths. However, the models of Marley et al. (1999) and Sudarsky et al. (2005) indicate that the albedo drops significantly in the range between $1.0 \mu \mathrm{m}$ and $1.2 \mu \mathrm{m}$ in a manner which is relatively independent of the star-planet separation. In the case of Jupiter, the effective temperature of Jupiter beyond $\sim 5 \mu \mathrm{m}$ is $\sim 125 \mathrm{~K}$ and the spherical albedo is $\sim 60 \%$ of the geometric albedo. In addition, constraints placed upon the spherical albedos of hot Jupiters (e.g., Knutson et al. 2009a) show these to be exceptionally low and in agreement with models which predict the removal of reflective cloud layers in those extreme stellar flux regimes. We thus generalize the albedo dependence on star-planet separation adopted by Kane \& Gelino (2010) by scaling the relation for spherical albedos. However, here we are testing the conditions at periastron where the albedo will be minimum. This is justified by the low measured albedos mentioned above and the independent statistical verification by Cowan \& Agol (2011b) which favors low Bond albedos for small star-planet separations.

\subsection{Heat Redistribution Efficiency}

The small phase amplitude of HD $189733 \mathrm{~b}$ detected by Knutson et al. (2009a) indicates that this particular planet has very high heat redistribution efficiency caused by atmospheric advection which produces high-speed zonal winds which carry heat to the night side of the planet. These atmospheric patterns are highly model dependent (Barbieri et al. 2007) and poorly understood for planets in the short-period regime due to the small sample size and the complex interaction of planetary structure, composition, tidal effects, and incident flux. Longer period eccentric orbits such as HD 80606b will further exhibit time-dependent behavior depending upon the star-planet separation and pseudo-synchronized spin rotation (see Equation (42) of Hut 1981). A statistical study of 24 known transiting planets performed by Cowan \& Agol (2011b) found that there is expected to be a wide range in heat redistribution efficiencies. As described earlier, the star-planet separation is dominant over heat redistribution efficiency in determining the planetary 
effective temperature. However, given the uncertainties in planetary models, we consider the two extremes of $\eta=0$ and $\eta=1$ to determine the upper and lower bounds on the flux ratio for a given planet.

\subsection{Radiative and Advective Time Constants}

The radiative time constant is a quantitative measure of the seasonal lag caused by the thermal response of the atmosphere to incident flux (Fortney et al. 2008; Seager et al. 2005). The radiative time constant, $\tau_{\text {rad }}$, is related to fundamental atmospheric properties in the following way:

$$
\tau_{\mathrm{rad}} \sim \frac{P}{g} \frac{c_{P}}{4 \sigma T_{p}^{3}},
$$

where $P$ is the pressure, $g$ is the surface gravity, and $c_{P}$ is the specific heat capacity. If $\tau_{\text {rad }}=0$ then the incident flux is immediately re-radiated from the day side of the planet (Cowan \& Agol 2011a). For example, the measured value for HD 80606 b by Laughlin et al. (2009) is $\tau_{\text {rad }}=4.5 \pm 2 \mathrm{hr}$.

The related quantity is the advective time constant, $\tau_{\mathrm{adv}}$, which is a measure of the movement of a parcel of gas around the planet. This is given by

$$
\tau_{\mathrm{adv}} \sim \frac{R_{P}}{U}
$$

where $U$ is the characteristic wind speed. Thus, $\tau_{\mathrm{adv}}$ approximates to zero when the wind speed becomes large, a situation which results in a high heat redistribution efficiency, as discussed in Section 3.2. The predicted values of $\tau_{\text {rad }}$ and $\tau_{\text {adv }}$ are highly dependent upon the circulation models of the atmospheres (Langton \& Laughlin 2008; Montalto et al. 2011; Showman et al. 2009) and also vary with the wavelength since this effects the depth to which the atmosphere is probed (Knutson et al. 2009a). Here, we concern ourselves with the peak flux ratio which is expected to occur relatively close to the point of periastron passage for eccentric orbits. Thus we assume that $\tau_{\text {rad }} \ll P$ and $\tau_{\text {adv }} \gg P$ for the subsequent calculations, keeping in mind that significant planetary spin, in addition to atmospheric composition and cloud effects, may induce wind patterns that cause divergence from this assumption. This produces an upper limit on the flux from the planetary substellar point and thus an upper limit on the predicted flux ratio with the star. The interplay between the radiative/advective time constants and other effects, such as drag mechanisms and numerical dissipation, have been investigated by Rauscher \& Menou (2011) and Thrastarson \& Cho (2011).

\section{FLUX RATIO AT PERIASTRON PASSAGE}

The measurable quantity from observations acquired at frequency $v$ is the flux ratio between the star and the planet, given by

$$
\frac{F_{p}}{F_{\star}}=\frac{\left(\exp \left(h v / k T_{\mathrm{eff}}\right)-1\right) R_{p}^{2}}{\left(\exp \left(h v / k T_{p}\right)-1\right) R_{\star}^{2}},
$$

where $R_{p}$ is the radius of the planet. The flux ratio as a function of the orbital phase depends upon the thermal redistribution efficiency of the atmosphere. If this is $100 \%$ efficient then the flux depends purely on the star-planet separation since there is no longer a phase function such as that described by Kane \& Gelino (2010).
Observations of HD 189733b and HD 80606b indicate that these planets have relatively high redistribution efficiency (Knutson et al. 2009a; Laughlin et al. 2009). These planets are in very different kinds of orbits and so this high efficiency may be quite common among planets which experience either constant or intermittent periods of high stellar flux. The lack of atmospheric measurements, and thus models to explain observations, for eccentric planets further motivates the need for this study. An alternative hypothetical explanation is that hot Jupiters have high zonal winds and very little cloud layer, whereas eccentric planets are not irradiated in the same way and so retain some of their clouds, even during periastron passage, thus reducing the redistribution efficiency (Cowan \& Agol 2011a). This could lead to phase variations in the thermal signature such that planets whose periastron argument is $\omega \sim 270^{\circ}$ become the optimal targets. Discriminating between these competing ideas requires further observations to resolve. The calculations in the following sections thus represent testable assumptions which can constrain these postulates.

\section{HABITABILITY OF ECCENTRIC PLANETS}

The HZ is defined as the range of circumstellar distances from a star within which a planet could have liquid water on its surface, given a dense enough atmosphere. The various criteria for defining the HZ have been described in detail by Kasting et al. (1993) and further generalized as a function of spectral type by Underwood et al. (2003) and Jones \& Sleep (2010). In estimating the boundaries of the HZ, we utilize the equations of Underwood et al. (2003) which relate the radii of the HZ inner and outer edges to the luminosity and effective temperature of the host star. Using the boundary conditions of runaway greenhouse and maximum greenhouse effects at the inner and outer edges of the HZ, respectively (Underwood et al. 2003), the stellar flux at these boundaries are given by

$$
\begin{gathered}
S_{\text {inner }}=4.190 \times 10^{-8} T_{\text {eff }}^{2}-2.139 \times 10^{-4} T_{\text {eff }}+1.268 \\
S_{\text {outer }}=6.190 \times 10^{-9} T_{\text {eff }}^{2}-1.319 \times 10^{-5} T_{\text {eff }}+0.2341 .
\end{gathered}
$$

The inner and outer edgers of the $\mathrm{HZ}$ are then derived from the following:

$$
\begin{gathered}
r_{\text {inner }}=\sqrt{L_{\star} / S_{\text {inner }}} \\
r_{\text {outer }}=\sqrt{L_{\star} / S_{\text {outer }}},
\end{gathered}
$$

where the radii are in units of $\mathrm{AU}$ and the stellar luminosities are in solar units.

The effect of orbital eccentricity on the habitability of planets has been investigated by Atobe et al. (2004) and Dressing et al. (2010). Here, we expand upon this by using our effective temperature calculations to determine the amount of time a planet spends in the HZ. There are two types of HZ situations we consider: planets in eccentric orbits that remain within the bounds of the $\mathrm{HZ}$ and planets in eccentric orbits that cross the boundaries of the HZ. In the latter case, the atmospheric relaxation timescales (governed by $\tau_{\text {rad }}$ and $\tau_{\text {adv }}$, described in Section 3.3) may moderate temperatures even during dramatic changes in star-planet separation. Note that long-period planets in circular orbits are more likely to have a non-synchronous spin rotation and so $\tau_{\text {rad }}$ and $\tau_{\text {adv }}$ will have a much smaller correlation with orbital period. However, planets in eccentric orbits will likely spend the majority of their orbits within the $\mathrm{HZ}$ if apastron lies close to the outer edge, thus retaining the 


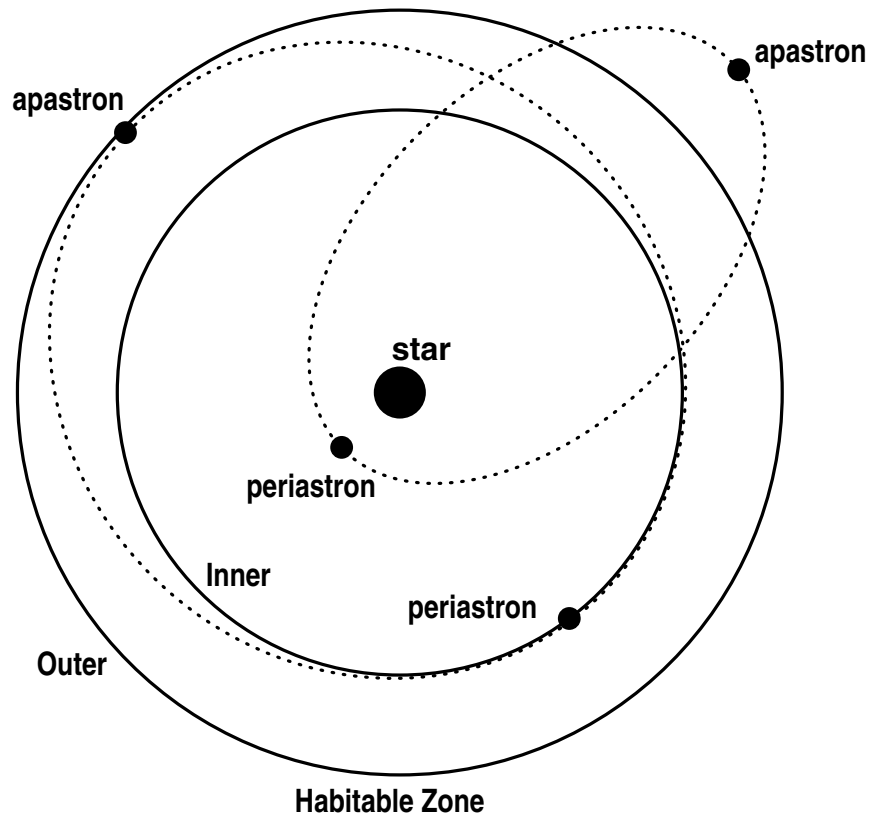

Figure 2. Eccentric orbits (dotted lines) relative to the inner and outer boundaries of the habitable zone (solid lines). The low eccentricity planet is able to maintain a presence inside the habitable zone, while the high eccentricity planet moves beyond both boundaries.

possibility of pseudo-synchronous spin rotation. Note that it is poorly understood to what degree exoplanets in eccentric orbits will retain a pseudo-synchronous spin rotation within the $\mathrm{HZ}$ due to the many contributing factors. However, the evidence for these relative influences has been observed for moons within our own solar system (Gladman et al. 1996). Figure 2 shows two example eccentric orbits overlaid on a hypothetical HZ which depicts the two cases described above. In the following section, we apply these methods to determine the percentage of the orbit spent in the HZ for the known exoplanets.

\section{APPLICATION TO KNOWN EXOPLANETS}

Here we calculate predicted effective temperatures and flux ratios at periastron passage for a sample of the known exoplanets for which there are known orbital solutions. The orbital parameters of 390 planets, along with the host star properties, were extracted using the Exoplanet Data Explorer ${ }^{1}$ (Wright et al. 2011). The data are current as of 2011 January 17. Planets for which the host star values of $T_{\text {eff }}$ and $\log g$ were not available were excluded from the sample. The calculated flux ratios are highly dependent on the assumed radii of the exoplanets (see Equation (9)). The anomalous radii of short-period transiting planets have been investigated by Laughlin et al. (2011). However, Fortney et al. (2007) have shown that, for a given planetary mass and composition, planetary radii should not vary substantially between orbital radii of 0.1-2.0 AU. However, different compositions, particularly with regards to core versus coreless models, can lead to radii variations of $\sim 25 \%$. Since we are considering eccentric orbits of massive planets, we take the conservative approach of fixing the radius for each of the planets in this sample at one Jupiter radius, keeping the caveats mentioned above in mind.

There are a variety of current and future space-based observatories that are capable of effectively monitoring known

\footnotetext{
http://exoplanets.org/
}

exoplanets for thermal signatures. The Infrared Array Camera (IRAC) instrument of the Spitzer Space Telescope had passbands centered at 3.6, 4.5, 5.8, and $8.0 \mu \mathrm{m}^{2}$ For the $J W S T$, planned instruments include MIRI which covers 5-27 $\mu \mathrm{m}^{3}$ and NIRCam which covers $0.6-5.0 \mu \mathrm{m} .{ }^{4}$ We calculate predicted flux ratios using the 3.6 and $4.5 \mu \mathrm{m}$ passbands of IRAC since the longer wavelengths are no longer available during the warm phase of the mission.

Table 1 shows the results of these calculations for the 50 most eccentric planets in the sample. Included in the table are the orbital period, $P$, eccentricity, $e$, periastron argument, $\omega$, the percentage of a full orbit spent in the $\mathrm{HZ}, t_{\mathrm{HZ}}$, and the effective temperatures and IR flux ratios assuming both low $(\eta=0)$ and high $(\eta=1)$ heat redistribution efficiencies. We also assume an albedo of $A=0.0$, meaning that the planet absorbs $100 \%$ of the incident flux (see Madhusudhan \& Seager 2009 for a more thorough statistical analysis of these values). This produces a higher effective temperature for the planet but is a reasonable assumption since models and measurements have shown that planets lose their reflective cloud layers at small star-planet separations (Kane \& Gelino 2010; Sudarsky et al. 2005). For comparison, an albedo of $A=0.3$ leads to a $\sim 10 \%$ reduction in the planetary effective temperatures. Note that flux ratios for those planets at particularly long periods are approximately zero at both passbands. However, recall from Figure 1 that the flux ratios are mostly dependent upon the star-planet separation at periastron rather than $\eta$. Thus, as one increases the orbital period and decreases the eccentricity, the change in $\eta$ becomes less important (also demonstrated later in the top-right panel of Figure 3). The flux ratios calculated for HD 80606b are comparable to the $0.0010 \pm 0.0002$ values measured by Laughlin et al. (2009) at $8 \mu \mathrm{m}$ and indicate that there is moderate heat redistribution efficiency of the atmosphere in this case. Some of the more interesting examples are discussed in detail in Section 8.

The top two panels of Figure 3 plot the planetary effective temperatures and flux values for all 390 planets included in the sample, assuming $\eta=1$ and $\lambda=4.5 \mu \mathrm{m}$. The planetary temperature plot on the left shows a reasonably even distribution of temperatures for circular orbits, as one may expect. However, note that there is a downward trend for eccentricities $\lesssim 0.5$ since these planets tend to lie at a large semimajor axis. A second trend occurs in the opposite direction (eccentricities $\gtrsim 0.5$ ); the temperature increases as the eccentricity increases since this decreases the star-planet separation at periastron. The temperature plot maps to the flux ratio plot shown on the right, where the hot Jupiters can be seen clustered in the top left corner. The upward trend in temperatures toward higher eccentricities results in an equivalent upward trend in flux ratios. There is no decrease in flux ratio toward higher eccentricities. Thus, it is clear that the high eccentricity planets present viable opportunities to detect their thermal signature during periastron passage.

The percentage of the total orbital period spent within the $\mathrm{HZ}$ is calculated by first estimating the boundaries of the $\mathrm{HZ}$ (see Section 5) and then determining the star-planet separation at equal increments in time during a Keplerian orbit. It is not surprising that most of the eccentric planets spend less than half of their time within the HZ due to the large range of star-planet separations which occur during the orbit. The bottom two panels of Figure 3 show how the planets which spend some part of

\footnotetext{
2 http://ssc.spitzer.caltech.edu/irac/

3 http://www.stsci.edu/jwst/instruments/miri/

4 http://www.stsci.edu/jwst/instruments/nircam/
} 
Table 1

Effective Temperatures and IR Flux Ratios for Known Exoplanets

\begin{tabular}{|c|c|c|c|c|c|c|c|c|c|c|}
\hline \multirow[b]{2}{*}{ Planet } & \multirow[b]{2}{*}{$\begin{array}{c}P \\
\text { (days) }\end{array}$} & \multirow[b]{2}{*}{$e$} & \multirow[b]{2}{*}{$\begin{array}{l}\omega \\
\left(^{\circ}\right)\end{array}$} & \multirow[b]{2}{*}{$\begin{array}{l}t_{\mathrm{HZ}} \\
(\%)\end{array}$} & \multicolumn{3}{|c|}{$\eta=0$} & \multicolumn{3}{|c|}{$\eta=1$} \\
\hline & & & & & $\begin{array}{l}T_{p} \\
(\mathrm{~K})\end{array}$ & $\begin{array}{c}F_{p} / F_{\star}\left(10^{-3}\right) \\
(3.6 \mu \mathrm{m})\end{array}$ & $\begin{array}{c}F_{p} / F_{\star}\left(10^{-3}\right) \\
(4.5 \mu \mathrm{m})\end{array}$ & $\begin{array}{l}T_{p} \\
(\mathrm{~K})\end{array}$ & $\begin{array}{c}F_{p} / F_{\star}\left(10^{-3}\right) \\
(3.6 \mu \mathrm{m})\end{array}$ & $\begin{array}{c}F_{p} / F_{\star}\left(10^{-3}\right) \\
(4.5 \mu \mathrm{m})\end{array}$ \\
\hline HD 80606 b & 111.4 & 0.934 & 300.6 & 39.7 & 1838.2 & 1.5762187 & 1.9343872 & 1545.7 & 1.0012931 & 1.3134501 \\
\hline HD 20782 b & 585.9 & 0.925 & 147.0 & 22.8 & 1080.4 & 0.2262089 & 0.3613180 & 908.5 & 0.1109253 & 0.2016740 \\
\hline HD 4113 b & 526.6 & 0.903 & 317.7 & 23.4 & 946.0 & 0.1481725 & 0.2604596 & 795.5 & 0.0660831 & 0.1351611 \\
\hline HD $156846 b$ & 359.5 & 0.847 & 52.2 & 60.5 & 1098.9 & 0.0946147 & 0.1502021 & 924.0 & 0.0469136 & 0.0845516 \\
\hline HD 43197 b & 327.8 & 0.830 & 251.0 & 77.9 & 851.5 & 0.0808349 & 0.1547891 & 716.0 & 0.0330801 & 0.0751514 \\
\hline HD 28254 b & 1116.0 & 0.810 & 301.0 & 18.6 & 619.3 & 0.0077581 & 0.0209590 & 520.7 & 0.0022853 & 0.0078623 \\
\hline HD $45350 \mathrm{~b}$ & 963.6 & 0.778 & 343.4 & 15.0 & 529.9 & 0.0042552 & 0.0142493 & 445.6 & 0.0010208 & 0.0045419 \\
\hline HD 30562 b & 1157.0 & 0.760 & 81.0 & 18.4 & 543.9 & 0.0031772 & 0.0102831 & 457.4 & 0.0007908 & 0.0033750 \\
\hline HD 20868 b & 380.9 & 0.750 & 356.2 & 36.6 & 601.7 & 0.0139532 & 0.0385124 & 506.0 & 0.0039670 & 0.0140474 \\
\hline HD 37605 b & 54.2 & 0.737 & 211.6 & 0.0 & 1160.8 & 0.4103989 & 0.6223644 & 976.1 & 0.2106124 & 0.3596571 \\
\hline HD $222582 \mathrm{~b}$ & 572.4 & 0.725 & 319.0 & 30.6 & 567.1 & 0.0077235 & 0.0234816 & 476.9 & 0.0020345 & 0.0080621 \\
\hline HD 8673 b & 1634.0 & 0.723 & 323.4 & 16.6 & 487.9 & 0.0011613 & 0.0044675 & 410.3 & 0.0002465 & 0.0012917 \\
\hline HD 2039 b & 1120.0 & 0.715 & 344.1 & 15.0 & 456.8 & 0.0012679 & 0.0054234 & 384.1 & 0.0002422 & 0.0014417 \\
\hline HD 96167 b & 498.9 & 0.710 & 285.0 & 60.9 & 745.3 & 0.0126504 & 0.0276510 & 626.7 & 0.0045728 & 0.0121864 \\
\hline HD 86264 b & 1475.0 & 0.700 & 306.0 & 19.6 & 487.1 & 0.0010770 & 0.0041537 & 409.6 & 0.0002280 & 0.0011985 \\
\hline HAT-P-13 c & 428.5 & 0.691 & 176.7 & 68.9 & 667.8 & 0.0111062 & 0.0273530 & 561.5 & 0.0035730 & 0.0110002 \\
\hline HD 159868 b & 986.0 & 0.690 & 97.0 & 30.0 & 523.3 & 0.0019888 & 0.0067876 & 440.0 & 0.0004686 & 0.0021329 \\
\hline HD 17156 b & 21.2 & 0.682 & 121.9 & 0.0 & 1767.7 & 0.6833214 & 0.8563483 & 1486.4 & 0.4281503 & 0.5754836 \\
\hline 16 Cyg B b & 798.5 & 0.681 & 85.8 & 20.4 & 463.6 & 0.0016843 & 0.0069971 & 389.8 & 0.0003295 & 0.0018960 \\
\hline HD 89744 b & 256.8 & 0.673 & 195.1 & 0.0 & 907.5 & 0.0321474 & 0.0589155 & 763.1 & 0.0138748 & 0.0298084 \\
\hline HD 39091 b & 2151.0 & 0.641 & 330.2 & 11.9 & 330.3 & 0.0000443 & 0.0003698 & 277.8 & 0.0000045 & 0.0000592 \\
\hline HD $131664 \mathrm{~b}$ & 1951.0 & 0.638 & 149.7 & 11.1 & 322.1 & 0.0000384 & 0.0003408 & 270.9 & 0.0000037 & 0.0000521 \\
\hline HD 74156 b & 52.0 & 0.630 & 174.0 & 0.0 & 1235.3 & 0.2161417 & 0.3191066 & 1038.7 & 0.1150297 & 0.1895808 \\
\hline HD 44219 b & 472.3 & 0.610 & 147.4 & 73.9 & 552.5 & 0.0045355 & 0.0143141 & 464.6 & 0.0011534 & 0.0047790 \\
\hline HD 154672 b & 163.9 & 0.610 & 265.0 & 0.0 & 766.4 & 0.0358256 & 0.0760486 & 644.4 & 0.0133102 & 0.0342433 \\
\hline HD 16175 b & 990.0 & 0.600 & 222.0 & 23.8 & 419.6 & 0.0004756 & 0.0023800 & 352.8 & 0.0000784 & 0.0005626 \\
\hline HD $3651 \mathrm{~b}$ & 62.2 & 0.596 & 245.5 & 0.0 & 833.3 & 0.1188941 & 0.2310287 & 700.7 & 0.0477421 & 0.1105272 \\
\hline HD $171028 b$ & 550.0 & 0.590 & 304.0 & 54.7 & 623.3 & 0.0045460 & 0.0121809 & 524.1 & 0.0013496 & 0.0045978 \\
\hline HIP 2247 b & 655.6 & 0.540 & 112.2 & 25.4 & 353.8 & 0.0001572 & 0.0010949 & 297.5 & 0.0000185 & 0.0001980 \\
\hline HD 190228 b & 1136.1 & 0.531 & 101.2 & 40.0 & 420.4 & 0.0001656 & 0.0008165 & 353.5 & 0.0000274 & 0.0001936 \\
\hline CoRoT-10 b & 13.2 & 0.530 & 218.9 & 0.0 & 1123.2 & 0.6402574 & 0.9856290 & 944.5 & 0.3219422 & 0.5607959 \\
\hline HD 142022 b & 1928.0 & 0.530 & 170.0 & 8.3 & 273.5 & 0.0000047 & 0.0000650 & 230.0 & 0.0000003 & 0.0000071 \\
\hline HD 87883 b & 2754.0 & 0.530 & 291.0 & 0.0 & 193.4 & 0.0000000 & 0.0000010 & 162.6 & 0.0000000 & 0.0000000 \\
\hline HD 108147 b & 10.9 & 0.530 & 308.0 & 0.0 & 1828.5 & 0.7475883 & 0.9266767 & 1537.6 & 0.4740451 & 0.6283575 \\
\hline HD 168443 b & 58.1 & 0.529 & 172.9 & 0.0 & 959.0 & 0.1119503 & 0.1943817 & 806.4 & 0.0504508 & 0.1016838 \\
\hline HD 81040 b & 1001.7 & 0.526 & 81.3 & 20.3 & 325.6 & 0.0000604 & 0.0005207 & 273.8 & 0.0000059 & 0.0000813 \\
\hline HIP 5158 b & 345.7 & 0.520 & 252.0 & 49.8 & 425.7 & 0.0012029 & 0.0057508 & 358.0 & 0.0002036 & 0.0013882 \\
\hline HD $148156 b$ & 1027.0 & 0.520 & 35.0 & 24.9 & 350.4 & 0.0001130 & 0.0008256 & 294.6 & 0.0000131 & 0.0001469 \\
\hline HD $217107 \mathrm{c}$ & 4270.0 & 0.517 & 198.6 & 0.0 & 197.0 & 0.0000000 & 0.0000008 & 165.6 & 0.0000000 & 0.0000000 \\
\hline HAT-P-2 b & 5.6 & 0.517 & 185.2 & 0.0 & 2498.6 & 1.2828619 & 1.4573069 & 2101.0 & 0.8890871 & 1.0564045 \\
\hline HD 1237 b & 133.7 & 0.511 & 290.7 & 42.5 & 585.8 & 0.0179584 & 0.0520765 & 492.6 & 0.0049349 & 0.0184897 \\
\hline HD 142415 b & 386.3 & 0.500 & 255.0 & 72.3 & 487.3 & 0.0023210 & 0.0088971 & 409.8 & 0.0004917 & 0.0025685 \\
\hline HD $215497 \mathrm{c}$ & 567.9 & 0.490 & 45.0 & 35.6 & 376.9 & 0.0002662 & 0.0016269 & 317.0 & 0.0000358 & 0.0003268 \\
\hline HD $106252 b$ & 1531.0 & 0.482 & 292.8 & 17.1 & 302.4 & 0.0000157 & 0.0001642 & 254.3 & 0.0000013 & 0.0000222 \\
\hline HD 33636 b & 2127.7 & 0.481 & 339.5 & 4.1 & 262.2 & 0.0000024 & 0.0000372 & 220.5 & 0.0000001 & 0.0000037 \\
\hline HD $181433 d$ & 2172.0 & 0.480 & 330.0 & 0.0 & 221.7 & 0.0000002 & 0.0000057 & 186.4 & 0.0000000 & 0.0000004 \\
\hline HD $196885 b$ & 1333.0 & 0.480 & 78.0 & 35.9 & 366.0 & 0.0000877 & 0.0005804 & 307.8 & 0.0000111 & 0.0001112 \\
\hline HD 33283 b & 18.2 & 0.480 & 155.8 & 0.0 & 1503.7 & 0.3606475 & 0.4817197 & 1264.5 & 0.2118062 & 0.3083335 \\
\hline HD $210277 \mathrm{~b}$ & 442.2 & 0.476 & 119.1 & 44.6 & 398.9 & 0.0005740 & 0.0031455 & 335.5 & 0.0000862 & 0.0006903 \\
\hline HD 154857 b & 409.0 & 0.470 & 59.0 & 32.1 & 575.2 & 0.0021955 & 0.0065333 & 483.7 & 0.0005893 & 0.0022767 \\
\hline
\end{tabular}

Note. The last six columns are for the cases of $\eta=0$ and $\eta=1$, respectively.

their orbit within the $\mathrm{HZ}$ are distributed according to period and eccentricity. The left plot shows the percentage of the orbit spent in the $\mathrm{HZ}, t_{\mathrm{HZ}}$, as a function of period. Interestingly, the planets which spend more than $20 \%$ of the orbit within the $\mathrm{HZ}$ are evenly distributed between $20 \%$ and $80 \%$ and orbital periods of 200-2000 days. To clarify the relative distributions of the planets which spend a non-zero amount of time within the HZ, we show the eccentricity of the planets as a function of period in the right plot. The relative size of the points increases as a function of the percentage time spent within the HZ. The planets which spend a portion of their time in the $\mathrm{HZ}$ are fairly evenly distributed in eccentricity, although the more circular orbits preferentially spend a greater percentage of their time there. This indicates that eccentricity can sometimes be a useful discriminator in selecting targets for potential life-bearing planets, although there are some relatively large points shown for $e>0.5$. In both of the bottom 

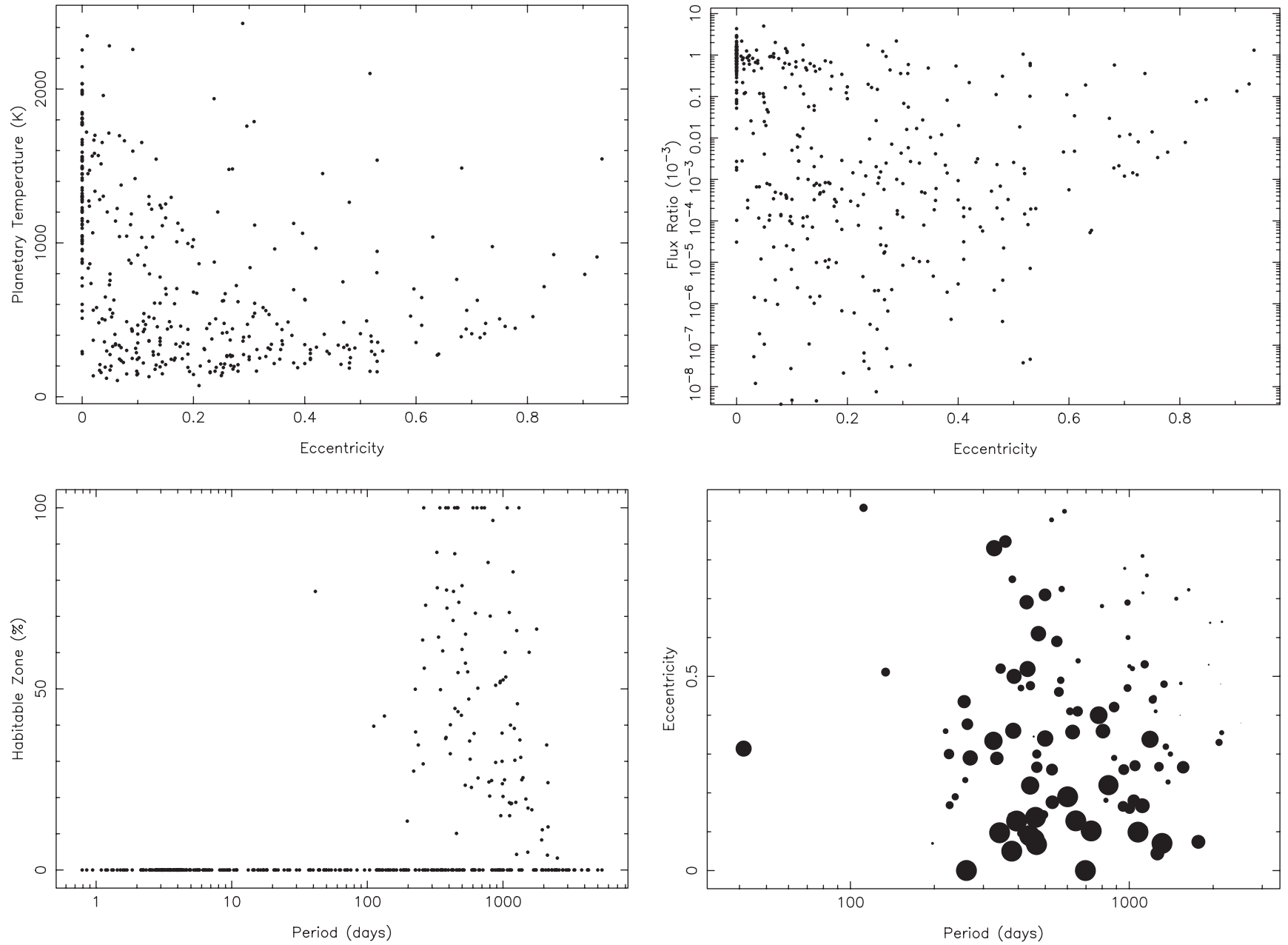

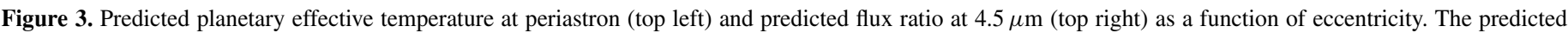

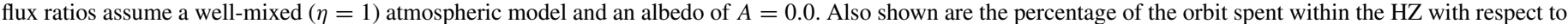

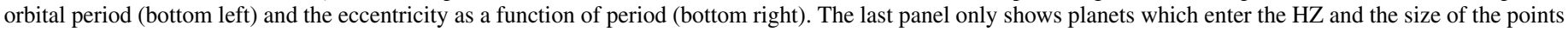
linearly increases with the percentage of time spent within the HZ.

plots, the outlier located at 41.4 days is the Saturn-mass planet orbiting the M4 dwarf HIP 57050, discovered by Haghighipour et al. (2010). Despite the relatively short period and eccentricity of 0.31 , the smaller luminosity of the host star allows this planet to spend most of the orbit within the HZ.

In Figure 4 we show two particularly interesting cases. On the left is shown the highly eccentric planet orbiting HD 43197. This planet spends $\sim 78 \%$ of the total orbital period residing within the HZ, although even considering $\eta=1$, the temperature rises to $716 \mathrm{~K}$ during periastron passage. On the right is shown the rather more benign orbit of the planet orbiting HD 156411. This planet is not listed in Table 1 (due to its relatively low eccentricity) but it spends $96.5 \%$ of the orbit within the confines of the $\mathrm{HZ}$ and reaches a peak temperature of $307 \mathrm{~K}$ during periastron passage assuming $\eta=1$. With a flux ratio of $7.8 \times 10^{-8}$ at $4.5 \mu \mathrm{m}$, one cannot reasonably expect to detect such a planet with current instrumentation, but it does present an interesting case for habitability studies of eccentric orbits. These two planets perfectly represent the two cases described in Figure 2.

\section{IMPACT OF STAR SPOTS ON DETECTION}

Since most of the known exoplanets we are concerned with here orbit F-, G-, or K-type main-sequence stars, we need to investigate the effect, if any, that star spots will have on the detection of these planets and their possible phase signatures in the IR. Berdyugina (2005) showed that, on average, the difference between the stellar photosphere and a stellar spot is larger for hotter stars with values near $2000 \mathrm{~K}$ for late-F and early-G stars and drops to $200 \mathrm{~K}$ for late-M stars. IR observations detect stars at cooler temperatures than optical observations, and therefore star spots will be observed at a lower contrast than in the optical. This means that, even though late-type stars typically have a greater number of star spots, these should be less of a flux contrast issue for the late-type stars that are better suited for IR observations.

Of concern is the timescale of star spot modulations compared with the timescale over which significant flux variation is expected to occur during periastron passage. As shown by Kane \& Gelino (2010), the maximum flux amplitude for eccentric planets is a small fraction of the total orbit and in many cases allows a $24-48 \mathrm{hr}$ window through which the maximum changes can be observed. In contrast, the rotation periods of typical exoplanet hosting stars are substantially longer. Rotation periods have been measured for many of these, such as the work of Henry et al. (1997) and Simpson et al. (2010). These rotation periods are mostly in the range of $20-40$ days, which is common of 1-5 Gyr old main-sequence stars. 

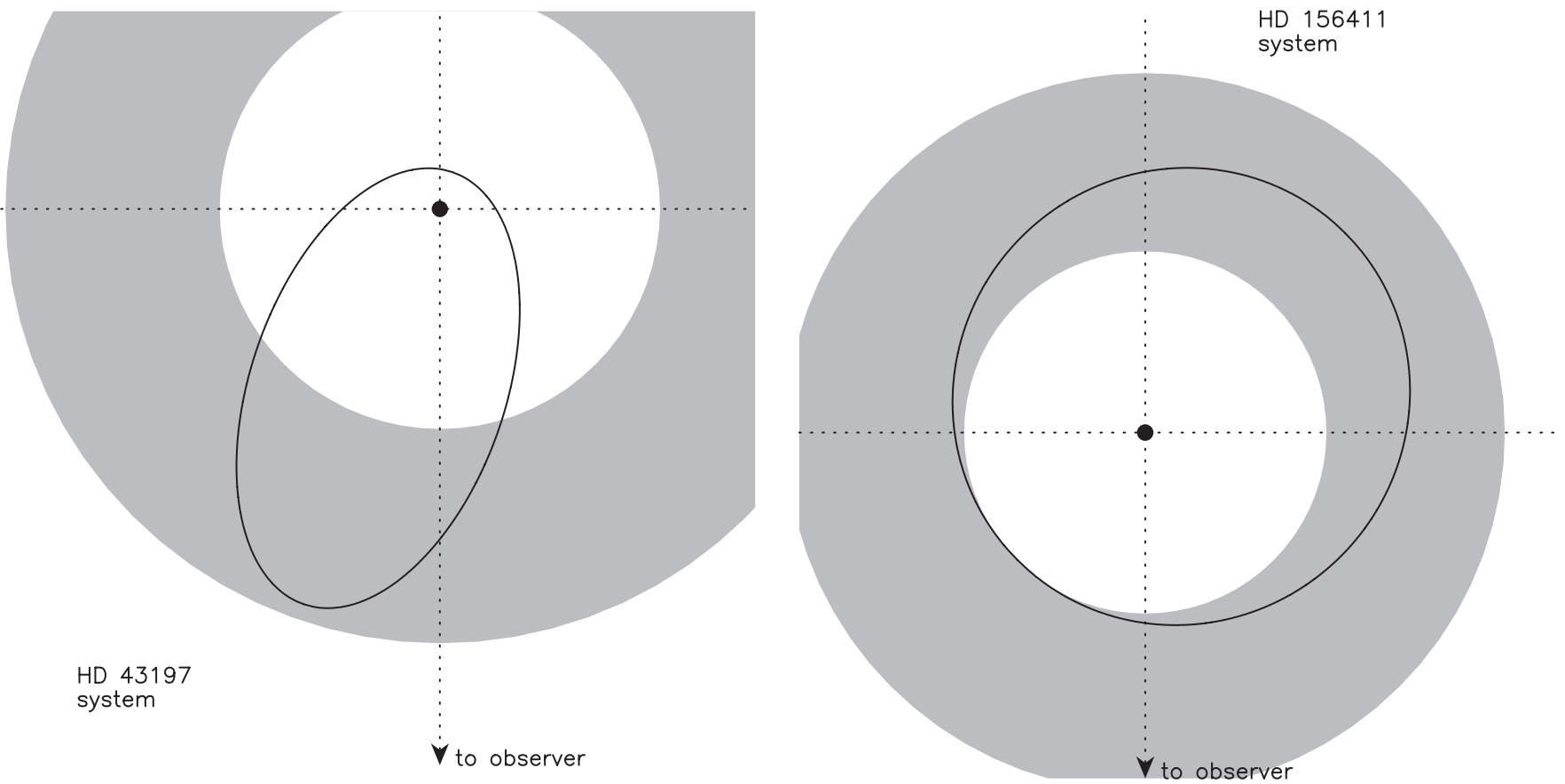

Figure 4. Habitable zones (shaded region) and orbits of the planets orbiting HD 43197 (left) and HD 156411 (right).

In the case of the Sun, star spots typically cover between $10^{-3}$ (during a solar maximum) and $10^{-5}$ of the surface. However, up to $22 \%$ of a hemisphere was seen to be covered in a Doppler image of XX Tri, which is a $\mathrm{K} 0$ giant and therefore not typical of exoplanet host stars (Strassmeier 2009). Star spots are likely to evolve over timescales of a few stellar rotation cycles or even within one cycle. This supports the results of Hussain (2002) who found that spots on single main-sequence stars, at most, live for weeks.

The frequency of star spot occurrence increases for low-mass stars which, at the current epoch, comprise the minority of exoplanet host stars. As described above, most stellar rotation periods are much longer than the periastron passage timescales. Therefore, it is usually not necessary to worry about star spots inhibiting the detection of the phase signatures of the planets. Stellar spot signatures change over time, so in the very few cases where the timescales may be similar, it should be possible to disentangle the planet phase signature from the star spot signature. Consistent with our findings, an IR study conducted by Désert et al. (2011a) found that the variability due to spots is less than the predicted transit depths and with a longer period. Conversely, Désert et al. (2011b) find that they do have to take into account spots on HD 189733 and conclude that an estimation of the planet-to-star radius ratio should be associated with a corresponding stellar activity level. Thus, each experiment should address this stellar activity level issue on a case-by-case basis.

\section{CASE STUDIES}

In this section, we consider several interesting case studies from the results in the previous sections.

\subsection{The HD 156846 System}

The planet orbiting HD 156846 has recently been studied by Kane et al. (2011), providing refined orbital parameters and the exclusion of additional giant planets in the system. Thus, even though the orbital period is large $(P=359$ days $)$, observations during periastron passage could be timed with great precision in order to detect a thermal signature. However, the periastron argument of $\omega=51^{\circ}$ means that the periastron passage occurs close to the observer-star line of sight on the near side of the star. Thus the night side of the planet faces toward us during periastron passage. This presents an opportunity to test the heat redistribution efficiency models for this planet since a low efficiency will result in a distinct phase function which prevents detection, but a high efficiency will still allow for a detectable signature as the heat is transferred to the night side of the planet.

\subsection{The HD 37605 and HD 33283 Systems}

Here we discuss the two interesting cases of HD $37605 \mathrm{~b}$ and HD 33283b. The flux ratio of HD $37605 \mathrm{~b}$ is second only to HD 80606b among the eccentric planets shown in Table 1, aided by the short orbital period compared with its large eccentricity. This planet was also suggested as an excellent candidate for phase monitoring at optical wavelengths by Kane \& Gelino (2010). The periastron argument of $\omega=211^{\circ}$ means that a significant fraction of the day side will be angled toward us during periastron passage. HD $33283 \mathrm{~b}$ has a very similar predicted flux ratio, the lower eccentricity being offset by a shorter orbital period. The host stars in both cases are bright enough such that a detection of phase variations during periastron passage will yield significant information regarding the atmospheric properties of the respective planets. A lack of detectable phase variation will in turn constrain atmospheric models for these planets regarding the mechanisms for which heat distribution is occurring.

Provided that the orbital parameters for these stars are sufficiently refined, the total time needed to achieve adequate coverage of the phase curves to maximize a detection is relatively low. Shown in Figure 5 are the calculated flux ratios at $4.5 \mu \mathrm{m}$ as a function of orbital phase for each of the planets. Zero orbital phase is defined to occur at superior conjunction and the location of the peak flux ratio depends upon the periastron argument of the orbit. This model assumes that the atmospheres 

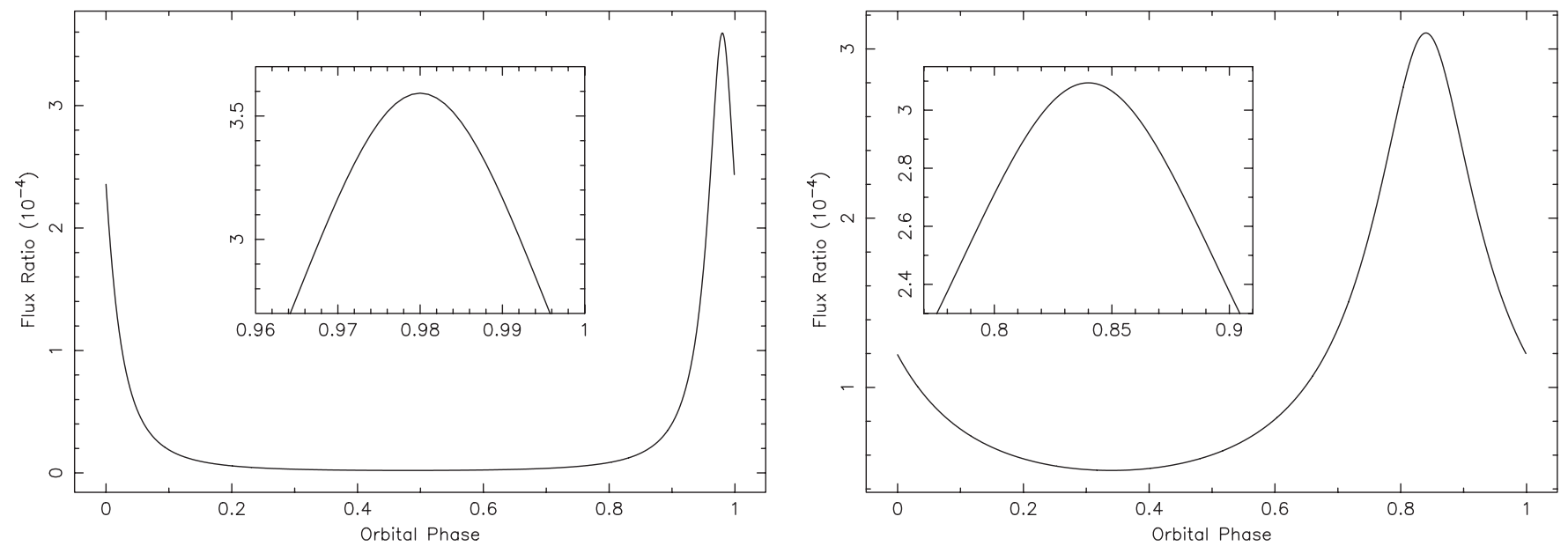

Figure 5. Phase curves for HD 37605b (left) and HD 33283b (right) at $4.5 \mu \mathrm{m}$, assuming $100 \%$ heat redistribution efficiency $(\eta=1)$ of the atmosphere. The sub-panels zoom in on the section of the phase curve which would be optimal for monitoring.

are very efficient at redistributing the heat $(\eta=1)$ which lowers the effective flux ratios. Thus, these models represent a minimum expected signature from each of the planets. Each of the figures contains a sub-panel which zooms in on the pericenter passage segment of the orbit. These segments can be adequately covered with $25 \mathrm{hr}$ of observations, therefore only $50 \mathrm{hr}$ in total would be needed to monitor both targets. The planets have the same time-coverage requirements because, although HD 37605 b has a longer period than HD 33283b, it also has a larger eccentricity which increases the planetary velocity at pericenter. Such targets represent rare opportunities to probe the atmospheric properties of planets beyond the regime of hot Jupiters and further develop theoretical models of these planets.

\section{CONCLUSIONS}

We have presented an analysis of the known exoplanets to determine predicted temperatures and flux ratios. This exercise is clearly not meant to serve as an exhaustive modeling of the atmospheres for each of these planets, and indeed combining the models of Fortney et al. (2010) and Lewis et al. (2010) with IR data will present new and exciting opportunities for studying the atmospheres of eccentric planets. Rather, this is meant to serve as a guide toward which of the particularly eccentric exoplanets may serve as interesting targets for follow-up observations.

For planets which lie within the HZ of their host stars, eccentric planets may present additional opportunities for studying planetary atmospheres in these zones. HD 43197b and HD $156411 \mathrm{~b}$ are particularly interesting cases since they each spend a majority of the total orbital period within their respective HZs but vary dramatically in the temperature differences experienced throughout their entire orbits. Thus, these cases may be used to investigate the effects of eccentric orbits upon habitability. Based on the fact that most stellar rotation periods are much longer than the periastron passage timescales, and that star spots typically have a signature that is much less than that of a HZ planet at periastron passage, we conclude that spots are not a significant issue for this particular study. Due to the changing nature of star spot activity on a given star, long-term monitoring of the star could disentangle the planet phase signature.

We have presented several case studies from our analysis which serve as potentially interesting targets for detection during periastron passage. HD $156846 \mathrm{~b}$ is a good IR target for studies of the atmospheric properties of planets in extreme (highly eccentric) orbits. HD 37605b and HD 33283b are also good targets since they have bright host stars, relatively high predicted flux ratios, and brief periods of large changes in the flux ratio. Warm Spitzer is capable of monitoring some of these targets during the remaining lifetime of the mission. When this mission ceases operations, JWST will have instrumentation that is capable of continuing this study to even higher precision.

The authors thank Ian Crossfield, Gregory Laughlin, Nicolas Cowan, and Lisa Prato for several useful discussions. We also thank the anonymous referee, whose comments greatly improved the quality of the paper. This research has made use of the Exoplanet Orbit Database and the Exoplanet Data Explorer at exoplanets.org.

\section{REFERENCES}

Agol, E., Cowan, N. B., Knutson, H. A., et al. 2010, ApJ, 721, 1861 Atobe, K., Ida, S., \& Ito, T. 2004, Icarus, 168, 223

Barbieri, M., Alonso, R., Laughlin, G., et al. 2007, A\&A, 476, L13 Barman, T. S., Hauschildt, P. H., \& Allard, F. 2005, ApJ, 632, 1132 Berdyugina, S. V. 2005, Living Rev. Sol. Phys., 2,8

Cowan, N. B., \& Agol, E. 2011a, ApJ, 726, 82

Cowan, N. B., \& Agol, E. 2011b, ApJ, 729, 54

Cowan, N. B., Agol, E., \& Charbonneau, D. 2007, MNRAS, 379, 641

Crossfield, I. J., Hansen, B. M. S., Harrington, J., et al. 2010, ApJ, 723, 1436

Cubillos, P. E., Rojo, P., \& Fortney, J. J. 2011, A\&A, 529, 88

Deming, D., Richardson, L. J., \& Harrington, J. 2007, MNRAS, 378, 148

Désert, J., Bean, J., Miller-Ricci Kempton, E., et al. 2011a, ApJ, 731, L40

Désert, J., Sing, D., Vidal-Madjar, A., et al. 2011b, A\&A, 526, 12

Dobbs-Dixon, I., Cumming, A., \& Lin, D. N. C. 2010, ApJ, 710, 1395

Dressing, C. D., Spiegel, D. S., Scharf, C. A., Menou, K., \& Raymond, S. N. 2010, ApJ, 721, 1295

Fortney, J. J., Lodders, K., Marley, M. S., \& Freedman, R. S. 2008, ApJ, 678, 1419

Fortney, J. J., Marley, M. S., \& Barnes, J. W. 2007, ApJ, 659, 1661

Fortney, J. J., Shabram, M., Showman, A. P., et al. 2010, ApJ, 709, 1396

Gladman, B., Dane Quinn, D., Nicholson, P., \& Rand, R. 1996, Icarus, 122, 166

Haghighipour, N., Vogt, S. S., Butler, R. P., et al. 2010, ApJ, 715, 271

Harrington, J., Hansen, B. M., Luszcz, S. H., et al. 2006, Science, 314, 623

Henry, G. W., Baliunas, S. L., Donahue, R. A., Soon, W. H., \& Saar, S. H. 1997, ApJ, 474, 503

Hussain, G. A. J. 2002, Astron. Nachr., 323, 349

Hut, P. 1981, A\&A, 99, 126

Jones, B. W., \& Sleep, P. N. 2010, MNRAS, 407, 1259

Kane, S. R., \& Gelino, D. M. 2010, ApJ, 724, 818

Kane, S. R., \& Gelino, D. M. 2011, ApJ, 729, 74

Kane, S. R., Howard, A. W., Pilyavsky, G., et al. 2011, ApJ, 733, 28

Kane, S. R., Mahadevan, S., von Braun, K., Laughlin, G., \& Ciardi, D. R. 2009, PASP, 121, 1386 
Kasting, J. F., Whitmire, D. P., \& Reynolds, R. T. 1993, Icarus, 101, 108 Knutson, H. A., Charbonneau, D., Cowan, N. B., et al. 2009a, ApJ, 690, 822 Knutson, H. A., Charbonneau, D., Cowan, N. B., et al. 2009b, ApJ, 703, 769 Koskinen, T. T., Aylward, A. D., Smith, C. G. A., \& Miller, S. 2007, ApJ, 661, 515

Langton, J., \& Laughlin, G. 2008, ApJ, 674, 1106

Laughlin, G., Crismani, M., \& Adams, F. C. 2011, ApJ, 729, L7

Laughlin, G., Deming, D., Langton, J., et al. 2009, Nature, 457, 562

Lewis, N. K., Showman, A. P., Fortney, J. J., et al. 2010, ApJ, 720, 344

Madhusudhan, N., \& Seager, S. 2009, ApJ, 707, 24

Madhusudhan, N., \& Seager, S. 2010, ApJ, 725, 261

Marley, M. S., Gelino, C., Stephens, D., Lunine, J. I., \& Freedman, R. 1999, ApJ, 513,879

Montalto, M., Santos, N. C., Boisse, I., et al. 2011, A\&A, 528, L17

Naef, D., Latham, D. W., Mayor, M., et al. 2001, A\&A, 375, L27
Rauscher, E., \& Menou, K. 2010, ApJ, 714, 1334

Rauscher, E., \& Menou, K. 2011, ApJ, submitted (arXiv:1105.2321)

Seager, S., Richardson, L. J., Hansen, B. M. S., et al. 2005, ApJ, 632, 1122

Showman, A. P., Cooper, C. S., Fortney, J. J., \& Marley, M. S. 2008, ApJ, 682 559

Showman, A. P., Fortney, J. J., Lian, Y., et al. 2009, ApJ, 699, 564

Simpson, E. K., Baliunas, S. L., Henry, G. W., \& Watson, C. A. 2010, MNRAS, 408, 1666

Smalley, B. 2005, Mem. Soc. Astron. Ital. Suppl., 8, 130

Strassmeier, K. G. 2009, A\&AR, 17, 251

Sudarsky, D., Burrows, A., \& Hubeny, I. 2005, ApJ, 627, 520

Thrastarson, H. T., \& Cho, J.Y.-K. 2010, ApJ, 716, 144

Thrastarson, H. T., \& Cho, J.Y.-K. 2011, ApJ, 729, 117

Underwood, D. R., Jones, B. W., \& Sleep, P. N. 2003, Int. J. Astrobiol., 2, 289

Wright, J. T., Fakhouri, O., Marcy, G. W., et al. 2011, PASP, 123, 412 